\title{
Original Research \\ A decade of insertable cardiac monitors with remote monitoring in pediatric patients
}

\author{
Massimo S. Silvetti ${ }^{1, *}$, Ilaria Tamburri ${ }^{1}$, Luigina Porco ${ }^{1}$, Fabio A. Saputo ${ }^{1}$, \\ Corrado Di Mambro ${ }^{1}$, Daniela Righi ${ }^{1}$, Ilaria Cazzoli ${ }^{1}$, Marianna Cicenia ${ }^{1}$, Marta Campisi ${ }^{1}$, \\ Lucilla Ravà $^{2}$, Cecilia Pizzicaroli ${ }^{3}$, Fabrizio Drago ${ }^{1}$ \\ ${ }^{1}$ Pediatric Cardiology and Cardiac Arrhythmias Unit, Bambino Gesù Children's Hospital IRCCS, 000165 Rome, Italy \\ ${ }^{2}$ Epidemiology Institute, Epidemiology Unit, Bambino Gesù Children's Hospital IRCCS, 000165 Rome, Italy \\ ${ }^{3}$ Anesthesiology Department Bambino Gesù Children's Hospital IRCCS, 000165 Rome, Italy \\ *Correspondence: mstefano.silvetti@opbg.net (Massimo S. Silvetti) \\ Academic Editor: Yoshiaki Kaneko \\ Submitted: 24 September 2021 Revised: 21 October 2021 Accepted: 28 October 2021 Published: 17 January 2022
}

\begin{abstract}
Background: Remote monitoring-enabled insertable cardiac monitors (ICMs) are useful tools for arrhythmias and symptom management. This study sought to evaluate the outcome of ICM implantation in a large, heterogeneous cohort of pediatric and young adult patients. Methods: Single centre, retrospective analysis of patients who underwent ICM implantation in 2010-2019. Patients were analysed according to age, symptoms, arrhythmias and underlying heart disease. Results: A total of 200 consecutive patients (58\% male), aged $11.5 \pm 5.8$ years at ICM implantation, were included. Follow-up was $31 \pm 18$ months. Electrophysiologic study (EPS) was initially performed in 123 patients and was negative in $85 \%$. Patients had no heart disease (57.5\%), congenital heart defects (21\%), channelopathies (14.5\%), cardiomyopathies/heart tumors (8\%). The commonest symptoms were syncope/presyncope (45.5\%) and palpitations (12.5\%). A definite diagnosis was made in $63 \%$ of patients (positive diagnosis in $25 \%$, negative in $38 \%$ ) after 8 (2-19) months of monitoring. EPS results and the presence/absence of an arrhythmia before ICM implantation had no impact on the diagnostic yield. Symptomatic patients as well as patients without structural heart disease showed higher diagnostic yield. Patients with a positive diagnosis underwent pacemaker/implantable cardioverter-defibrillator implantation (13\%), pharmacological treatment (10.5\%), or catheter ablation (1.5\%). Conclusions: In a large cohort of 200 children and young adults, ICMs with remote monitoring showed a high diagnostic yield (63\%), especially in symptomatic patients and in patients without structural heart disease.
\end{abstract}

Keywords: Insertable cardiac monitors; Remote monitoring; Syncope; Pediatric age; Congenital heart defects; Inherited arrhythmia

\section{Introduction}

An insertable cardiac monitor (ICM), or implantable loop recorder, is a device implanted in the chest subcutaneous tissues. It can be activated either automatically or by the patient/family member to store the electrocardiogram (ECG) recorded during an event [1]. The latest generation devices enable remote monitoring and facilitate early diagnosis by allowing daily automatic and patient-activated transmissions [2].

ICMs can play an important role in the detection of rare arrhythmias and in the diagnostic workup of syncope [3] and palpitations [4].

This study sought to evaluate the outcome of ICM implantation in a large, heterogeneous cohort of pediatric and young adult patients.

\section{Methods}

All consecutive patients who underwent ICM implantation between January 2010 and December 2019 were included in the study.

This study complies with the Declaration of Helsinki and was approved by the local ethics committee. Informed consent was obtained from all patients or their guardians.

All patients underwent ICM implantation after a comprehensive non-invasive evaluation $[5,6]$ including clinical visit, standard 12-lead ECG, Holter monitoring, exercise test, external loop recorder and imaging studies as appropriate. Additionally, an electrophysiologic study (EPS) was often performed in patients with suspected tachyarrhythmias or when the evaluation of the sinus node and atrioventricular node-His bundle conduction was required [7]. In patients $<15 \mathrm{~kg}$ of weight, transesophageal EPS was performed by applying the same protocol when only atrial pacing was needed.

\subsection{Procedure}

The implant procedure has previously been reported in detail [8] and is described below briefly. Most procedures were performed under general anesthesia or deep sedation, except for older adolescents and in adults, who underwent local anesthesia. The implantation site was guided by surface mapping to determine ECG signal quality and R-wave sensing. Pediatric ECG patches were placed at a $4 \mathrm{~cm}$ dis- 
tance (the width of device electrodes) on the patient skin in the desired ICM location and connected to a Medtronic Pacing System Analyzer. The implantation site was considered adequate when recorded signals had the following characteristics: R wave $>0.3 \mathrm{mV}$, R wave amplitude at least twice the amplitude of $\mathrm{P}$ and $\mathrm{T}$ waves. A $1 \mathrm{~cm}$ cut was made in the skin and the device was inserted over the muscular fascia using the provided insertion tool. Antibiotic prophylaxis (cefuroxime) was given at the beginning of the procedure. Patients were discharged the day after implantation according to Institutional protocols. months.

In-hospital follow-up was scheduled every 6-12

\subsection{Device programming}

Device programming was personalized according to symptoms or type of heart disease and patients were reprogrammed from out-of-box factory settings if needed. In general, bradycardia detection was set at $30 \mathrm{bpm}$ ( 4 beats), pauses at $3.0 \mathrm{~s}$, and tachycardia at 180-200 bpm (8-12 beats) in older children and adolescents. In younger children ( $<6$ years), the upper rate limit was $210-220$ bpm (1224 beats) and the lower rate limit was $40 \mathrm{bpm}$. In general, in adults with congenital heart defects (CHD) the limits were 150-180 bpm for tachycardia and $30 \mathrm{bpm}$ for bradycardia.

Atrial tachycardia/fibrillation detection was generally programmed off.

In patients with catecholaminergic polymorphic ventricular tachycardia (CPVT), tachycardia detection was set at $30 \%$ over the maximum sinus rate without arrhythmias at exercise test.

All patients received the "Medtronic CareLink" remote monitoring system.

\subsection{Diagnostic criteria}

A positive diagnosis was made when symptomatic tachy- or brady-arrhythmias or relevant asymptomatic arrhythmias were detected. Relevant asymptomatic arrhythmias included: advanced/complete atrio-ventricular block (AVB), ventricular pause $>3 \mathrm{~s}$, rapid ( $>180 \mathrm{bpm}$ ) prolonged/sustained paroxysmal supraventricular (SVT) or ventricular tachycardia (VT) [3].

A negative diagnosis was defined as no rhythm abnormalities during symptoms.

When a positive diagnosis was made, the patient underwent in-hospital clinical assessment and therapy. In case of a negative arrhythmic diagnosis, patients and families were reassured by phone.

\subsection{Statistical analysis}

Categorical data were expressed by counts and percentages. Continuous data were expressed by mean and standard deviation if normally distributed, or by median and interquartile range (25th-75th percentile) if nonnormally distributed. Normality was tested with the
Kolmogorov-Smirnov test. Patients were divided into subgroups according to symptoms (syncope/presyncope; syncope/presyncope and palpitations; palpitations; no symptoms; other symptoms) and age (infants and small children [aged 1-5 years], children [6-11 years], adolescents [12-18 years], adults [ $>18$ years]). Syncope and presyncope were also analysed separately. The definitions of syncope and presyncope followed current guideline criteria [3].

Patients were also divided according to arrhythmias and heart disease known prior to ICM implantation. Long QT syndrome (LQTS), Brugada syndrome, VT, SVT, advanced/3rd degree AVB, and sinus node dysfunction (SND) were considered as severe arrhythmias; premature ventricular complex (PVC), 1st and 2nd degree Mobitz 1 AVB, and ventricular preexcitation as not severe. For statistical purpose, SND and sinus pauses were considered in the same group. Patients' parameters were compared according to age category, symptoms, heart disease, arrhythmias, using Chi squared test or Fisher exact test for categorical variables and Student's $t$-test or ANOVA for continuous variables. A $p$-value $<0.05$ was considered statistically significant.

Statistical analysis was performed using STATA 14.1 software (Stata Corp., College Station, TX, USA).

\section{Results}

\subsection{Demographics}

The study included 200 patients (Table 1 ). There were 33 patients aged $1-5$ years, 69 patients aged $6-11$ years, 73 patients aged $12-18$ years, and 25 adult patients with CHD, aged $21.6 \pm 3.7$ years.

\subsection{Electrophysiologic study}

The EPS was performed in 123 patients before ICM implantation (14 transesophageal and 109 intracavitary EPS). In the majority of cases (85\%), EPS was negative. In the remaining $15 \%$, EPS showed inducibility of nonsustained atrial flutter (4 patients) or fibrillation ( 2 patients), non-sustained VT (4 patients), non-sustained SVT (5 patients), prolonged sinus node recovery time ( 2 patients) and prolonged $\mathrm{H}-\mathrm{V}$ interval (1 patient). However, these findings were considered non-specific and/or did not lead to a definite diagnosis or treatment. For example, patients with prolonged sinus node recovery time and $\mathrm{H}-\mathrm{V}$ interval were correctly identified, but there was no clear indication for pacemaker implantation. Thus, in patients with nonspecific findings (positive EPS), the ICM was implanted for further monitoring and ultimate diagnosis.

\subsection{Symptoms/Arrhythmias/Heart diseases}

Symptoms and arrhythmias leading to ICM implantation are shown in Table 1. The characteristics of the 38 patients with AVB are reported in Table 2. Patients with complete AVB underwent ICM implantation to record heart rate, and to exclude pauses and ventricular arrhythmias. Four of the five patients with complete AVB showed ven- 
Table 1. Demographic and pre-implantation data $(\mathrm{n}=\mathbf{2 0 0})$

\begin{tabular}{|c|c|}
\hline \multicolumn{2}{|l|}{ Characteristics } \\
\hline Male sex & $117(58.5)$ \\
\hline Female sex & $83(41.5)$ \\
\hline Age, years & $11.5 \pm 5.8$ \\
\hline Weight, kg & $45 \pm 21$ \\
\hline Height, $\mathrm{cm}$ & $144 \pm 26$ \\
\hline \multicolumn{2}{|l|}{ Heart disease } \\
\hline No heart disease & $115(57.5)$ \\
\hline Congenital heart defects* & $42(21)$ \\
\hline Channelopathy* & $29(14.5)$ \\
\hline Cardiomyopathy*, cardiac tumour & $16(8)$ \\
\hline \multicolumn{2}{|l|}{ Arrhythmias } \\
\hline Atrio-ventricular block & $38(19)$ \\
\hline Sinus node dysfunction, pauses & $17(8.5)$ \\
\hline Supraventricular tachycardia & $23(11.5)$ \\
\hline Ventricular tachycardia & $23(11.5)$ \\
\hline Premature ventricular complex & $19(9.5)$ \\
\hline Long QT syndrome & $13(6.5)$ \\
\hline Ventricular preexcitation & $9(4.5)$ \\
\hline Brugada syndrome & $6(3)$ \\
\hline No arrhythmia & $52(26)$ \\
\hline \multicolumn{2}{|l|}{ Symptoms } \\
\hline Syncope/presyncope & $91(45.5)$ \\
\hline Palpitations & $25(12.5)$ \\
\hline Syncope/presyncope + palpitations & $43(21.5)$ \\
\hline No symptoms & $33(16.5)$ \\
\hline Other & $8(4)$ \\
\hline $\begin{array}{l}\text { Values are given as } \mathrm{n}(\%) \text { or mean } \pm \\
\text { patient had both cardiomyopathy and lo } \\
\text { one had both cardiomyopathy and cong } \\
\text { symptoms include dyspnoea and asthen }\end{array}$ & $\begin{array}{l}\text { deviation. }{ }^{*} \text { One } \\
\text { yndrome, another } \\
\text { eart defect. Other } \\
\text { e. }\end{array}$ \\
\hline
\end{tabular}

tricular pauses $>$ three-fold the cycle length of the underlying rhythm and severe bradycardia during junctional escape rhythm and underwent pacemaker implantation (see below). Asymptomatic patients (16.5\%) underwent ICM implantation because of known arrhythmias or heart disease requiring monitoring (e.g., channelopathy). Heart diseases are shown in Tables 1 and 3.

\subsection{Implantation procedure}

The devices implanted were Medtronic Reveal XT $9529^{\mathrm{TM}}(\mathrm{n}=17$, implanted in the years 2010-2013), and Medtronic Reveal LINQ LNQ11 ${ }^{\mathrm{TM}}$ ( $\mathrm{n}=183$, the new miniaturized ICM implanted between 2013 and 2019).

The devices were implanted in the subcutaneous precordial (198 cases) or axillary (2 small children) pocket (Fig. 1).

Sensing at implantation was $1.3 \pm 0.8 \mathrm{mV}$ (range 0.4 $5.4 \mathrm{mV})$.
Table 2. Patients with atrio-ventricular block.

\begin{tabular}{|c|c|c|c|}
\hline Arrhythmia & No. & Symptoms & ICM diagnosis \\
\hline \multicolumn{4}{|l|}{ Low-degree AVB } \\
\hline 1st degree & 7 & \multirow{2}{*}{11} & \multirow{2}{*}{$77 \%$ (negative diagnosis $62 \%$ ) } \\
\hline 2nd degree Mobitz 1 & 6 & & \\
\hline \multicolumn{4}{|l|}{ High-degree AVB } \\
\hline Advanced $(2: 1,3: 1)$ & 7 & \multirow{3}{*}{13} & \multirow{3}{*}{$64 \%$ (positive diagnosis $44 \%$ ) } \\
\hline Paroxysmal 3rd degree & 13 & & \\
\hline Complete 3rd degree & 5 & & \\
\hline Total & 38 & 24 & \\
\hline
\end{tabular}

(A)

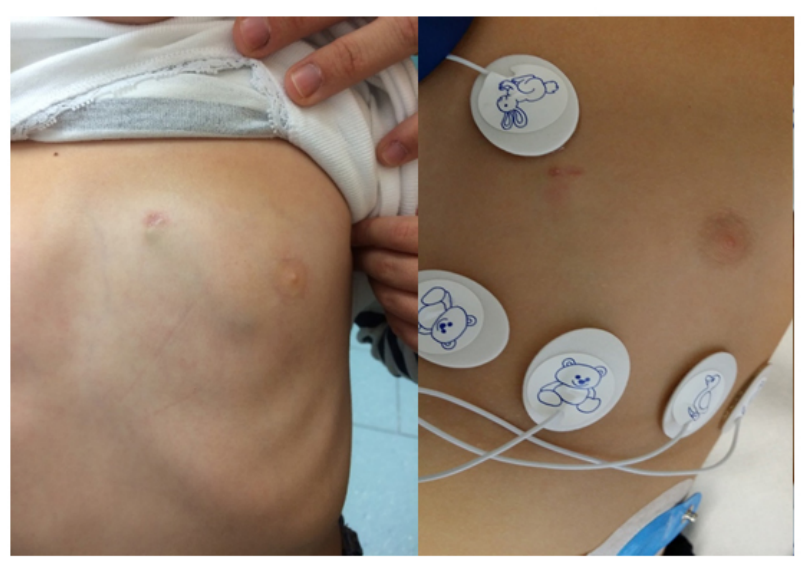

(B)

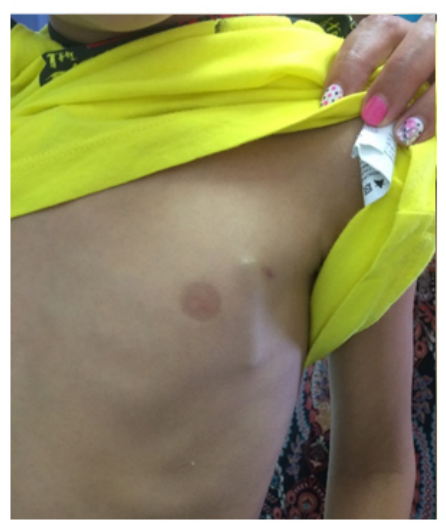

Fig. 1. Miniaturized ICMs implanted in children. ICMs implanted in children: precordial (A) and axillary (B) subcutaneous pockets.

\subsection{Follow-up}

Follow-up extended to February 2020, and its overall duration was $31 \pm 18$ months [median 31 (15-42) months].

After reaching the end of battery life, ICMs were reimplanted in 32 patients to continue monitoring, because of undefined diagnosis. In this subgroup, total follow-up was $58 \pm 10$ months.

Complications (pocket erosion) were recorded in 6 patients $(3 \%)$ with a median age of $11(3-24)$ years and a me- 
Table 3. Heart diseases.

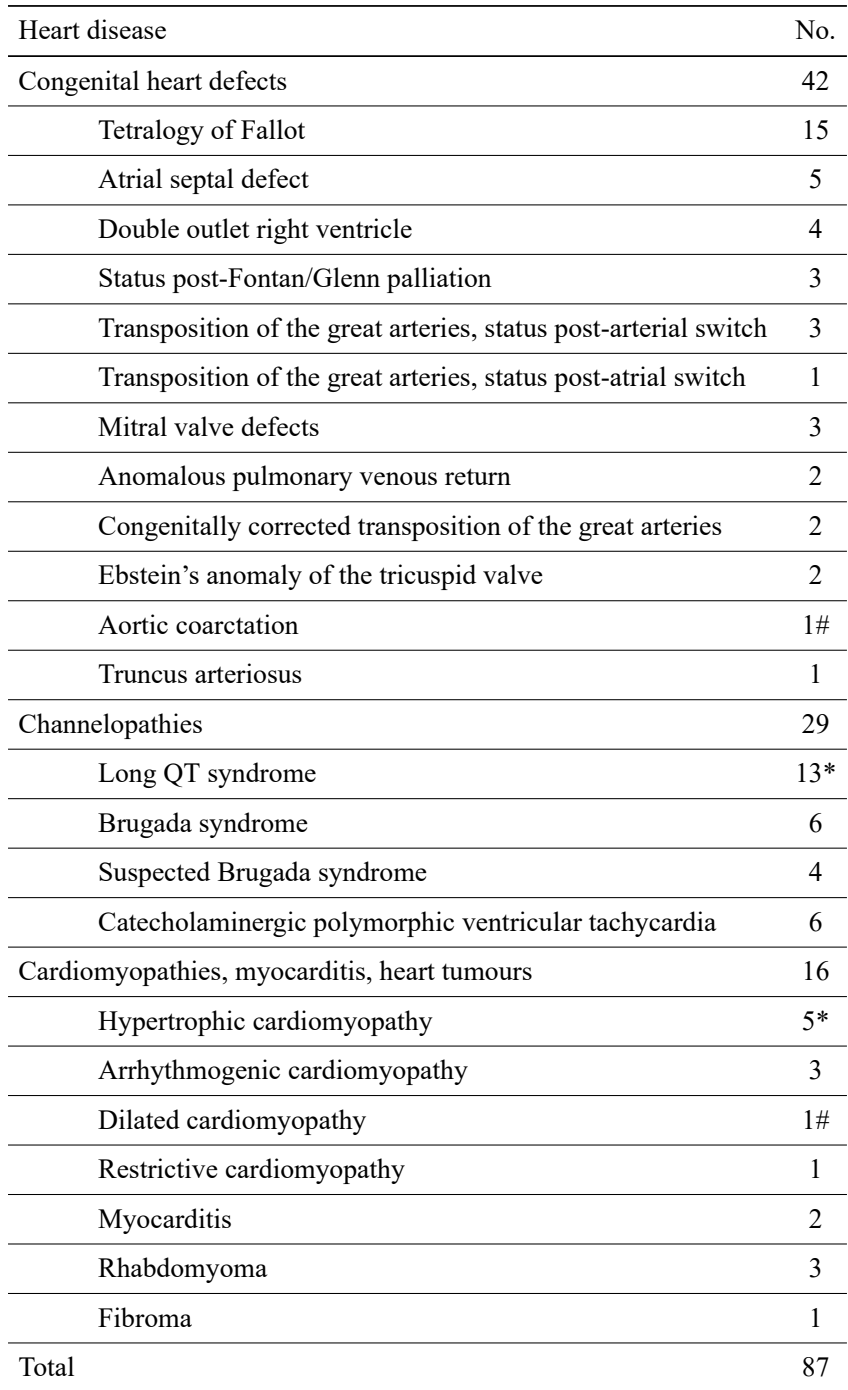

\#One patient had both cardiomyopathy and congenital heart defect.

*One patient had both cardiomyopathy and long QT syndrome.

dian weight of 42 (17-62) $\mathrm{kg}$ at ICM implantation. Erosions occurred within the first 3 months after implantation. The ICM was explanted in all cases.

\subsection{Outcome}

An arrhythmia diagnosis was made in 126 patients (63\%), and was positive in 50 patients $(25 \%)$ and negative in 76 patients $(38 \%)$. Time to diagnosis was $8(2-19)$ months. A diagnosis was not made in the remaining 74 patients $(37 \%)$. The events recorded were device-initiated in $54 \%$ of cases and patient-initiated in $46 \%$.

Patients with an ICM diagnosis were older (12.2 \pm 5.9 years) than those without a diagnosis $(10.4 \pm 5.5$ years) $(p$ $=0.036$ ). However, there were no significant differences according to age subgroups.

In the 32 patients who underwent ICM replacement, the diagnostic yield was $44 \%(n=14)$, with a positive diagnosis in $25 \%$ and a negative diagnosis in $19 \%$.

\subsection{Electrophysiologic study}

The EPS results had no impact on the diagnostic yield ( $p=0.6$ ). Among the 105 patients with a negative EPS, ICM monitoring was not diagnostic in 35 cases (33\%) and was diagnostic in the remaining $67 \%$. Among the 18 patients with a positive EPS, ICM monitoring was not diagnostic in 7 cases (39\%) and was diagnostic in the remaining $61 \%$.

\subsection{Symptoms}

Patients with symptoms leading to ICM implantation showed significantly higher diagnostic yields (89\%) than asymptomatic patients $(29 \% ; p=0.0001)$.

A significantly higher diagnostic rate was detected for palpitations $(76 \%)$ when compared to other symptoms ( $p$ $=0.0001$ ) (Fig. 2). Whenever syncope was differentiated from presyncope, it showed a higher diagnostic rate than presyncope: syncope $(70 \%)$, presyncope $(46 \%)$, syncope associated with palpitations $(80 \%)$, presyncope and palpitations $(69 \%)(p=0.0001)$.

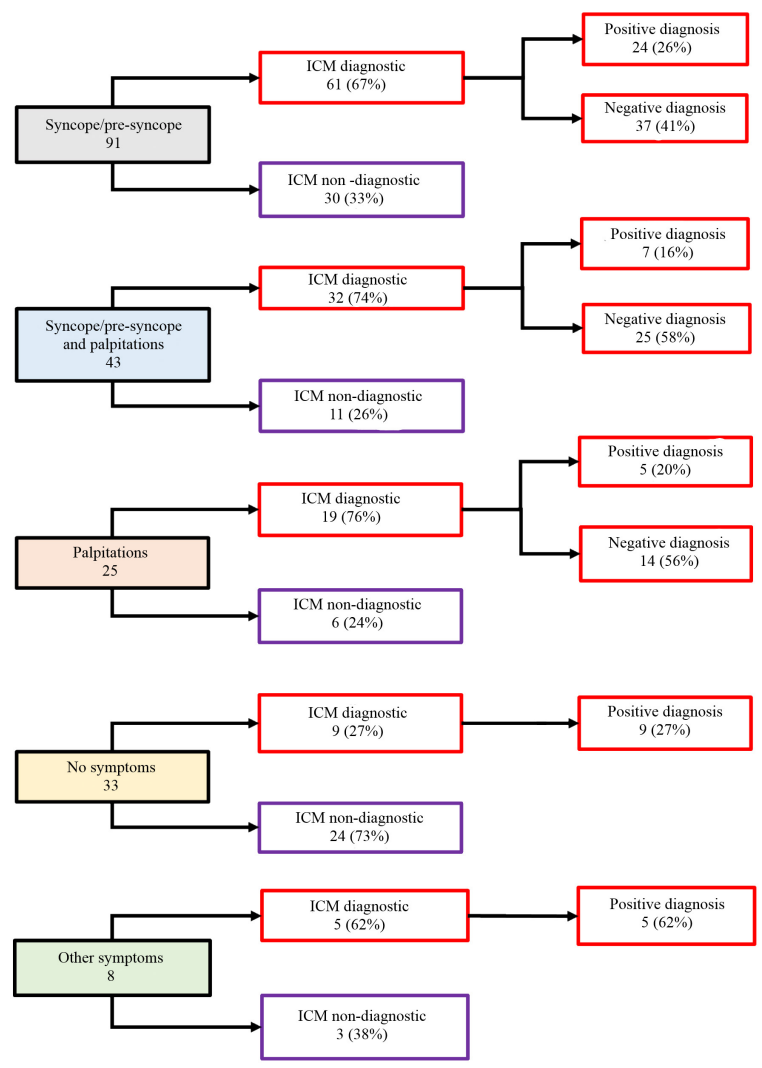

Fig. 2. ICM outcome. Diagram of ICM outcome according to patients' symptoms at implantation. $p=0.0001$.

\subsection{Arrhythmias}

The presence or absence of arrhythmias before ICM implantation (Table 1) was not significantly associated with a diagnosis defined by ICM, with a diagnostic yield of $61 \%$ vs $67 \%(p=0.3)$. 
Table 4. Outcome according to heart disease.

\begin{tabular}{lccc}
\hline Heart disease & No. patients & Diagnosis by ICM & No diagnosis by ICM \\
\hline No heart disease & 115 & $82(71 \%)$ & $33(29 \%)$ \\
\hline Congenital heart defects & 41 & $25(61 \%)$ & $16(39 \%)$ \\
\hline Channelopathies & 28 & $13(46 \%)$ & $15(54 \%)$ \\
\hline Cardiomyopathies/myocarditis & 10 & $4(40 \%)$ & $6(60 \%)$ \\
\hline Cardiomyopathy and channelopathy & 1 & 0 & $1(100 \%)$ \\
\hline Cardiomyopathy and congenital heart defect & 1 & $1(100 \%)$ & 0 \\
\hline Tumours & 4 & $1(25 \%)$ & $3(75 \%)$ \\
\hline Total & 200 & $126(63 \%)$ & $74(37 \%)$ \\
\hline Significant differences $(p=0.008)$. & & &
\end{tabular}

The analysis of arrhythmias already known at implantation showed that the diagnostic yield of ICMs was not significantly different: SVT (78\%), SND (76\%), AVB (63\%), PVC (58\%) and VT (39\%) $(p=0.09)$. The diagnostic outcome in AVB patients is reported in Table 2.

\subsection{Heart disease}

The outcome according to heart disease is reported in Table 4 and showed a significant difference $(p=0.008)$. Patients without structural heart disease showed the highest diagnostic yield, followed by those with CHD.

Only one patient with LQTS showed sustained VT at ICM monitoring and was effectively treated by antiarrhythmic drugs.

No relevant arrhythmias were recorded among the 6 patients with Brugada syndrome and the 4 patients with suspected Brugada syndrome.

The 6 patients with CPVT showed non-relevant arrhythmias or symptoms during ICM monitoring and continued their pharmacologic therapy.

\subsection{Device/drug treatment}

Pacemaker and implantable cardioverter-defibrillator implantation was performed in 22 and 4 patients, respectively. Cryoablation of slow pathway was performed in 3 patients. Drug treatment was started in 21 patients, using antiarrhythmic drugs in 10 patients and aminophylline in 11 patients with severe cardioinhibitory syncope.

\section{Discussion}

Since the new millennium, ICMs have been implanted in children and adolescents, with a diagnostic yield of 50$71 \%$ (9-13). ICMs have been found to be more diagnostic in symptomatic (94\%) than asymptomatic patients (30\%) [13]. However, a substantial number of symptoms remained unexplained [12]. Previous studies showed that complications (pocket erosion/infection) occurred in up to $7 \%$ of patients [10-13]. The longest median follow-up durations were $18-19$ months $[10,11]$.

Over the past decade, the new miniaturized ICMs have become available $[8,14]$. Placidi et al. [8] first described the results of this new devices implanted in very young and small children. In that study, overall diagnostic yield was $47 \%$ but pocket erosion/infections occurred in $9 \%$ of patients [8]. In 2019, Bezzerides et al. [15] described 133 patients with ICMs and found a diagnostic yield of $58 \%$, with positive diagnoses in $40 \%$ and negative diagnoses in $60 \%$ during a median follow-up of 12 months. Complications occurred in $4.5 \%$ of patients. Overall, $22 \%$ of patients received device treatment, $12 \%$ medication change and $6 \%$ EPS/ablation [15].

To the best of our knowledge, the current study reports the results of the largest cohort of pediatric and young adult ICM patients with the longest median follow-up (31 vs. 18 months [10]. The data obtained showed an overall diagnostic yield of $63 \%$, which was higher in symptomatic patients $(89 \%)$, in patients without structural heart disease $(71 \%)$ and in those with CHD (61\%). These findings are consistent with those reported for the pediatric population, with an overall diagnostic yield of $63 \%$ (vs. 58-67\%) $[9,12,15]$, a diagnostic yield in symptomatic patients of $89 \%$ (vs. 90\%) [13], and in those with syncope of $70 \%$ (vs. 72\%) [12]. The diagnostic yield was higher for patients with palpitations $(76 \%$ vs. $25-43 \%)[12,15]$. This finding may be due to the longer follow-up.

Negative arrhythmic diagnoses were generally more frequent than positive diagnoses, that is, symptoms occurred without any arrhythmia registered. Interestingly, in patients who underwent ICM replacement for further monitoring, the diagnostic yield was lower and positive diagnoses were more frequent than negative arrhythmic diagnoses. The main symptoms were syncope and palpitations, which are common symptoms in children and adolescents. Cardiac syncope occurred in 5\%, and undefined syncope in $3 \%$ of patients referred to a Pediatric Syncope Unit [16]. In the current study, symptomatic patients had a high diagnostic yield especially in the presence of palpitations. The diagnostic yield rose to $80 \%$ when patients had both syncope and palpitations. Conversely, presyncope showed a lower diagnostic yield. This result is new, not being reported in previous studies that included only few cases of near syncope $[9,12]$. 
(A)
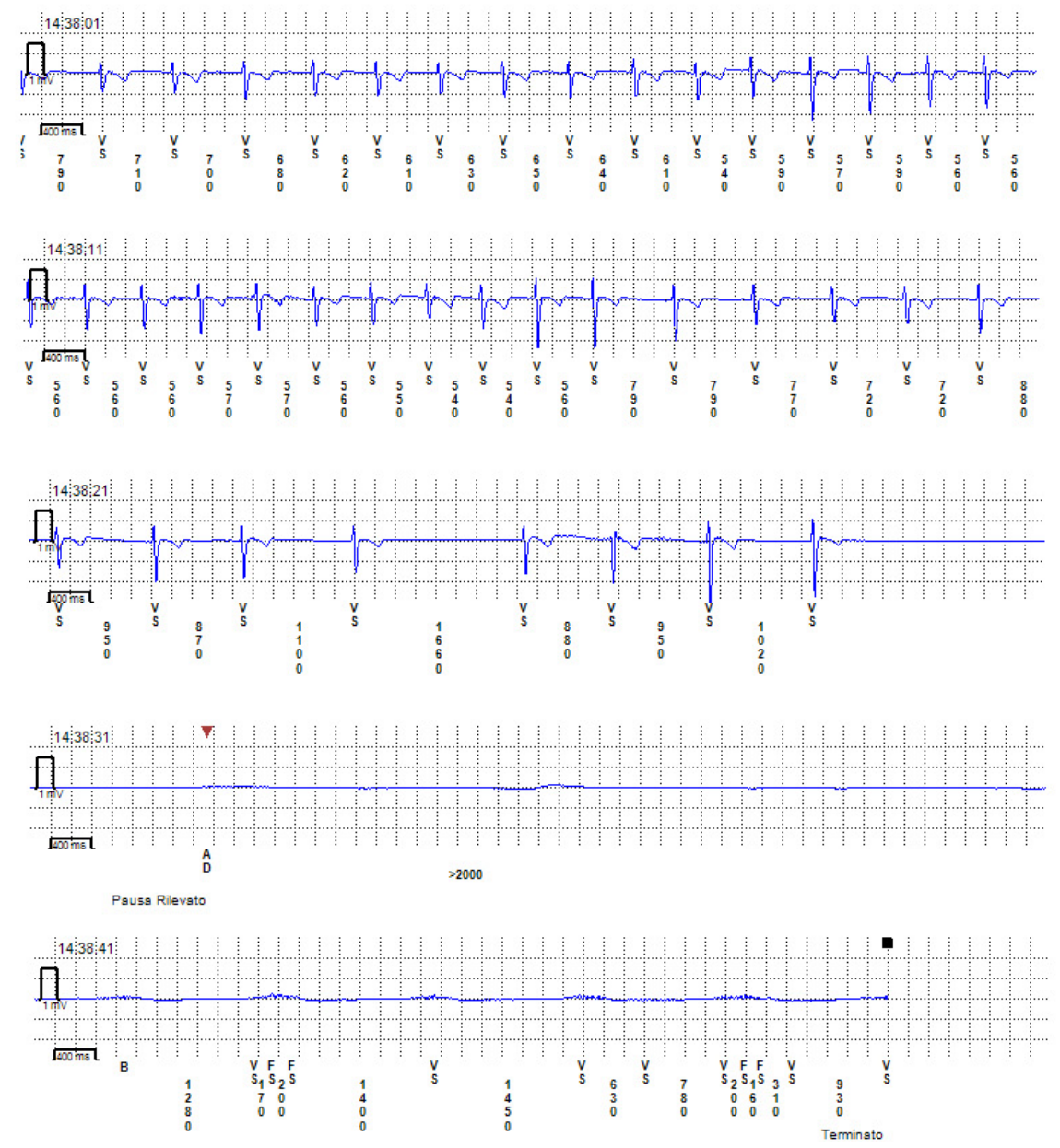

(B)
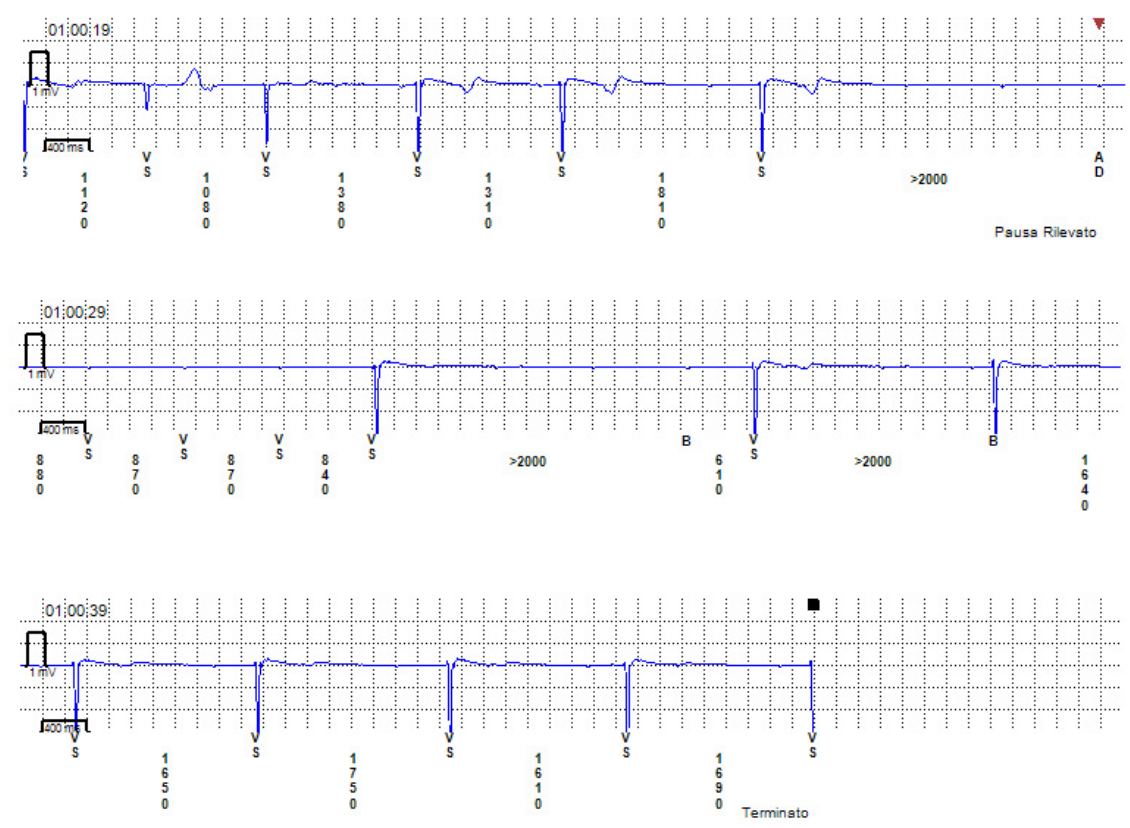

Fig. 3. ICM findings: oversensing. (A) Sinus arrest. Oversensing of noise, registered as ventricular activity (ventricular sensing, VS and fibrillation sensing, FS, that concluded the automatic detection of the pause, underestimating its duration). (B) 3rd degree AVB with long RR pauses. Rare oversensing of P waves detected as ventricular sensing (VS). 
Our previous data revealed a $100 \%$ diagnostic yield of ICM in children with palpitations and negative EPS [8]. Data from this study in a larger population followed up for a longer period confirmed that high diagnostic yield. A negative arrhythmic diagnosis was established in the majority of patients. A positive diagnosis was made in few patients and was mainly due to atrio-ventricular node reentry tachycardia, which should be considered in case of suspicious symptoms and when other examinations, including EPS, proved inconclusive [17].

The presence of arrhythmias leading to ICM implantation was not significantly associated with a higher diagnostic yield. However, bradyarrhythmias (AVB and SND/sinus pauses) and SVT showed the best results.

AVB was the main arrhythmia leading to implantation. Patients with high-degree AVB showed mainly positive diagnoses, most often leading to pacemaker implantation (Table 2). Patients with low-degree AVB showed mainly negative arrhythmic diagnoses, requiring no treatment. These findings show that a definite diagnosis and appropriate therapy could most often be established in patients with high-degree AVB, even if asymptomatic. In patients with low-degree AVB, in whom ICM was more often implanted than in high-degree AVB because of symptoms, negative diagnoses were frequent, thus excluding the need for therapy and allowing patient's reassurance. This is the case of symptomatic patients (syncope/presyncope, etc.) in whom ECG recording showed 1st degree or Mobitz 1 2nd degree AVB, but also unexpected asymptomatic (for example, nocturnal) 2:1, 3:1 or paroxysmal complete AVB. In these cases, ICM monitoring often showed that symptoms were not correlated with arrhythmias and these higher-degree AVB episodes did not require pacemaker implantation [18]. Conversely, episodes of symptomatic intermittent high-degree AVB led to pacemaker implantation.

The diagnostic performance was lower for PVCs and VT. In case of PVCs, this might occur because symptomatic patients with PVC had often symptoms not related to PVC, and monitoring did not show more severe arrhythmias. Furthermore, the Medtronic Reveal LINQ ${ }^{\mathrm{TM}}$ cannot automatically detect or count the PVC, but PVC episodes can be detected by patient-activated recording. On the contrary, in case of VT, ICM programming can justify the low diagnostic yield: if VT rate is lower than the tachycardia limit, asymptomatic episodes might be undetected. Conversely, if the tachycardia limit is too low, sinus tachycardia can saturate the ICM memory and the device might not record true tachycardias.

Sensing defects can occur as well. During asystolic pauses, ventricular oversensing (muscular movements, noise artifacts) may cause underestimation of pause durations (Fig. 3). A pause episode is detected by the device when the interval between the last ventricular complex before and the first ventricular complex after the pause exceeds the planned interval. The device terminates a pause episode when it records 12 ventricular markers following its detection. However, if there are further pauses in an episode without 12 ventricular markers in between, the device will consider only the duration of the first pause. The further pauses can be detectable on ECG but are not included in the duration counter. For this reason, patients/parents were instructed not only to register the symptom but also to make a patient-activated transmission as soon as possible after an episode.

Among structural heart disease, CHD patients showed the highest diagnostic yield (61\%), whereas those with cardiomyopathies the lowest. This finding seems quite consistent with that previously reported in pediatric and adult CHD patients (71\%) [10] and higher than that found in adult CHD patients (41\%) [19].

Conversely, channelopathies did not show a high diagnostic yield, as reported in adults [20]. Nowadays ICMs can be implanted in asymptomatic children with severe arrhythmias, including LQTS, CPVT, Brugada syndrome, or variants of unknown significance. This approach is new but justified by the small device dimensions and by the possibility of obtaining an early recognition of dangerous arrhythmias and rhythm monitoring during unclear symptoms. Therefore, this approach can guide management, and continuous cardiac monitoring may provide psychological protection to parents. However, it was shown that symptoms were not reliable surrogates for arrhythmia in children with inherited arrhythmia syndromes [21].

\section{Limitations}

This is a retrospective, single-centre study. Population selection might be biased by the fact that our institution is a third level centre and a Pediatric Syncope Unit, and acts as a referral centre for syncope/presyncope. This might result in higher proportions of patients with this symptom compared with a real-world scenario. Although the number of patients is quite large for a pediatric cohort, subgroup numbers may be too small for robust comparisons. Albeit some analyses were performed on the results of EPS and ICM monitoring, the study was not intended to compare or demonstrate a superiority of any of these procedures.

\section{Conclusions}

In a large pediatric population after long-term followup, ICMs showed a high diagnostic yield in the overall cohort, which was significantly higher in (1) symptomatic patients, mostly with palpitations, (2) patients without structural heart disease, and (3) patients with syncope rather than those with presyncope.

Patients with positive/negative diagnoses were significantly older than those without an arrhythmia diagnosis. However, there were no significant differences according to age subgroups.

Although most patients had negative EPS before implantation, ICMs were diagnostic in a high proportion of 
patients. The diagnostic yield was higher in CHD patients than in patients with cardiomyopathies and channelopathies. Prolonged monitoring after ICM replacement did not increase the diagnostic yield.

\section{Author contributions}

MSS and FD-methodology; MSS, FD, LP, CDM, DR, IC, MCI, CP, IT, FAS, MC-validation; MSS and FD-visualization; MSS, FD, LP, CDM, DR, IC, MCI, CP, LR, IT, FAS, MC - formal analysis, investigation and data curation; MSS, FD, LP, CDM, DR, IC, MCI, CP, LRwriting - original draft preparation; MSS, FD, LP, CDM, DR, IC, MCI, CP, LR — writing - review and editing.

\section{Ethics approval and consent to participate}

The study was conducted according to the guidelines of the Declaration of Helsinki, and approved by the Institutional Review as part of the Ricerca Corrente RRC-20202366948 .

\section{Acknowledgment}

The authors thank Elisa Del Vecchio for her valuable collaboration in the editorial revision.

\section{Funding}

This research received no external funding.

\section{Conflict of interest}

The authors declare no conflict of interest.

\section{References}

[1] Shen WK, Sheldon RS, Benditt DG, Cohen MI, Forman DE, Goldberger ZD, et al. 2017 ACC/AHA/HRS Guideline for the evaluation and management of patients with syncope: executive summary: a report of the American college of cardiology/American heart association task force on clinical practice guidelines and the heart rhythm society. Journal of The American College of Cardiology. 2017; 70: 620-663.

[2] Slotwiner D, Varma N, Akar JG, Annas G, Beardsall M, Fogel RI, et al. HRS Expert Consensus Statement on remote interrogation and monitoring for cardiovascular implantable electronic devices. Heart Rhythm. 2015; 12: e69-e100.

[3] Brignole M, Moya A, de Lange FJ, Deharo JC, Elliott PM, Fanciulli A, et al. 2018 ESC Guidelines for the diagnosis and management of syncope. European Heart Journal. 2018; 39: 18831948.

[4] Giada F, Gulizia M, Francese M, Croci F, Santangelo L, Santomauro M, et al. Recurrent unexplained palpitations (RUP) study. Journal of the American College of Cardiology. 2007; 49: 1951-1956.

[5] Brignole M, Vardas P, Hoffman E, Huikuri H, Moya A, Ricci $\mathrm{R}$, et al. Indications for the use of diagnostic implantable and external ECG loop recorders. Europace. 2009; 11: 671-687.

[6] Locati ET, Vecchi AM, Vargiu S, Cattafi G, Lunati M. Role of extended external loop recorders for the diagnosis of unexplained syncope, pre-syncope, and sustained palpitations. Europace. 2014; 16: 914-922.

[7] Josephson ME. Sinus Node Function. Clinical cardiac electrophysiology: techniques and interpretations. In: Josephson ME, edit. Malvern (PA), USA: Lea \& Febiger; 1993.

[8] Placidi S, Drago F, Milioni M, Verticelli 1, Tamburri I, Silvetti MS, et al. Miniaturized implantable loop recorder in small patients: an effective approach to the evaluation of subjects at risk of sudden death. Pacing and Clinical Electrophysiology. 2016; 39: 669-674.

[9] Rossano J, Bloemers B, Sreeram N, Balaji S, Shah MJ. Efficacy of implantable loop recorders in establishing symptom-rhythm correlation in young patients with syncope and palpitations. Pediatrics. 2003; 112: e228-e233.

[10] Kenny D, Chakrabarti S, Ranasinghe A, Chambers A, Martin R, Stuart G. Single-centre use of implantable loop recorders in patients with congenital heart disease. Europace. 2009; 11: 303 307.

[11] Kothari DS, Riddell F, Smith W, Voss J, Skinner JR. Digital implantable loop recorders in the investigation of syncope in children: benefits and limitations. Heart Rhythm. 2006; 3: 13061312.

[12] Al Dhahri KN, Potts JE, Chiu CC, Hamilton RM, Sanatani S. Are implantable loop recorders useful in detecting arrhythmias in children with unexplained syncope? Pacing and Clinical Electrophysiology. 2009; 32: 1422-1427.

[13] Frangini PA, Cecchin F, Jordao L, Martuscello M, Alexander $\mathrm{ME}$, Triedman $\mathrm{JK}$, et al. How revealing are insertable loop recorders in pediatrics? Pacing and Clinical Electrophysiology. 2008; 31: 338-343.

[14] Nguyen HH, Law IH, Rudokas MW, Lampe J, Bowman TM, Van Hare GF, et al. Reveal LINQ versus reveal XT implantable loop recorders: intra- and post-procedural comparison. The Journal of Pediatrics. 2017; 187: 290-294.

[15] Bezzerides VJ, Walsh A, Martuscello M, Escudero CA, Gauvreau K, Lam G, et al. The real-world utility of the LINQ implantable loop recorder in pediatric and adult congenital heart patients. JACC: Clinical Electrophysiology. 2019; 5: 245-251.

[16] Drago F, Calvieri C, Placidi S, Righi D, Paglia S, Del Vecchio $\mathrm{E}$, et al. Use of a pediatric syncope unit improves diagnosis and lowers costs: a hospital-based experience. The Journal of Pediatrics. 2018; 201: 184-189.e2.

[17] Raviele A, Giada F, Bergfeldt L, Blanc JJ, Blomstrom-Lundqvist $\mathrm{C}$, Mont L, et al. Management of patients with palpitation: a position paper from the European Heart Rhythm Association. Europace. 2011; 13: 920-934.

[18] Silvetti MS, Grutter G, Di Ciommo V, Drago F. Paroxysmal atrioventricular block in young patients. Pediatric Cardiology. 2004; 25: 506-512.

[19] Dodeja AK, Thomas C, Daniels CJ, Kertesz N, Kamp A. Detection of arrhythmias in adult congenital heart disease patients with LINQTM implantable loop recorder. Congenital Heart Disease. 2019; 14: 745-751.

[20] Sakhi R, Theuns DAMJ, Bhagwandien RE, Michels M, Schinkel AFL, Szili-Torok T, et al. Value of implantable loop recorders in patients with structural or electrical heart disease. Journal of Interventional Cardiac Electrophysiology. 2018; 52: 203-208.

[21] Avari Silva JN, Bromberg BI, Emge FK, Bowman TM, Van Hare GF. Implantable loop recorder monitoring for refining management of children with inherited arrhythmia syndromes. Journal of the American Heart Association. 2016; 5: e003632. 